\title{
Textbook of Pediatric Gastroenterology, Hepatology and Nutrition: Anupam Sibal, Sarath Gopalan (eds), Akshay Kapoor, Vidyut Bhatia (co-eds)
}

\author{
Published by Jaypee Brothers Medical Publishers (P) Ltd, New Delhi, India; First Edition: \\ 2015; ISBN: 978-93-5152-740-4
}

\author{
Nishant Wadhwa ${ }^{1}$
}

Received: 15 June 2015 / Accepted: 16 June 2015 /Published online: 7 July 2015

(C) Dr. K C Chaudhuri Foundation 2015

The first volume of the multi authored book titled "Textbook of Pediatric Gastroenterology Hepatology and Nutrition" edited by Dr. Anupam Sibal and Dr. Sarath Gopalan is a welcome addition to those who are keen to update their knowledge in Pediatric Gastroenterology. This book will be definitely beneficial in fine-tuning the diagnostic and managerial skills of budding subspecialists in their day-to-day practice. The book consists of 37 chapters on carefully assorted topics.

The initial 18 chapters are dedicated to Gastroenterology and Nutrition, a field that concerns the general pediatricians as much as it is of interest to those pursuing their studies in this specialty. The chapter on common gastrointestinal symptoms deserves a special mention as it is one of the most exhaustive and very informative one and should interest the gastroenterologist the most in his quest to decipher the symptomatology in pediatric gastrointestinal practice. The algorithms mentioned in various chapters aid in elucidating the approach to various disorders of the gut in a simple and lucid manner. Complex disorders of motility have been dealt with in a thought provoking manner in the subsequent chapter. The chapter on the surgical disorders is very well illustrated and should catch the interest of the clinician as much as it would

Nishant Wadhwa

docnish@gmail.com

1 Division of Pediatric Gastroenterology and Hepatology, Pediatric Liver Transplantation, Institute of Child Health, Sir Ganga Ram Hospital, New Delhi 110060, India interest the student of surgery. The section on nutrition encompasses nearly all aspects of parenteral and enteral nutrition along with the assessment of malnutrition in children. The chapter on food allergy beautifully describes certain case scenarios, which illustrates the practical utility of the principles discussed in a thought provoking manner.

The Hepatology section consists of 19 chapters covering both the acute and chronic liver diseases in reasonable detail. The chapter on drug-induced injury in children highlights this usually neglected area of pediatric Hepatology. There is an elaborate description of liver transplantation procedure including the indications and evaluation for the same. It also describes the pre and post transplant management principles in some detail.

There is no mention of interventional procedures (diagnostic and therapeutic) in pediatric gastrointestinal practice, several of which are central to the practice of this specialty in today's day and age. Since the book aims at fulfilling the needs of the young doctors training in the subspecialty it would have been worthwhile to have a chapter on interventional procedures. There is also no mention of the drugs and their uses, doses and side effects in both pediatric gastrointestinal and hepatology practice. The details of gastrointestinal pharmacopeia including doses and indications form an integral part of a textbook on such a subject. The editors will have to be more watchful in the preparation of the subsequent volumes of this book and hopefully include these areas too.

Notwithstanding these shortcomings, this textbook does provide a holistic and comprehensive view of the pediatric gastrointestinal and liver diseases and should be of use to both the general pediatrician and the followers of the sub-specialty. 